Wright State University

CORE Scholar

Kno.e.sis Publications

The Ohio Center of Excellence in Knowledge-

Enabled Computing (Kno.e.sis)

$11-2009$

\title{
Ontology-Driven Provenance Management in eScience: An Application in Parasite Research
}

\author{
Satya S. Sahoo \\ Wright State University - Main Campus \\ D. Brent Weatherly \\ Raghava Mutharaju \\ Wright State University - Main Campus \\ Pramod Anantharam \\ Wright State University - Main Campus \\ Amit P. Sheth \\ Wright State University - Main Campus, amit@sc.edu
}

See next page for additional authors

Follow this and additional works at: https://corescholar.libraries. wright.edu/knoesis

Part of the Bioinformatics Commons, Communication Technology and New Media Commons, Databases and Information Systems Commons, OS and Networks Commons, and the Science and

Technology Studies Commons

\section{Repository Citation}

Sahoo, S. S., Weatherly, D. B., Mutharaju, R., Anantharam, P., Sheth, A. P., \& Tarleton, R. L. (2009). OntologyDriven Provenance Management in eScience: An Application in Parasite Research. Lecture Notes in Computer Science, 5871, 992-1009.

https://corescholar.libraries.wright.edu/knoesis/669

This Conference Proceeding is brought to you for free and open access by the The Ohio Center of Excellence in Knowledge-Enabled Computing (Kno.e.sis) at CORE Scholar. It has been accepted for inclusion in Kno.e.sis Publications by an authorized administrator of CORE Scholar. For more information, please contact librarycorescholar@wright.edu. 


\section{Authors}

Satya S. Sahoo, D. Brent Weatherly, Raghava Mutharaju, Pramod Anantharam, Amit P. Sheth, and Rick L. Tarleton 


\title{
Ontology-driven Provenance Management in eScience: An Application in Parasite Research
}

Satya S. Sahoo $^{1}$, D. Brent Weatherly², Raghava Mutharajuㄹ, Pramod Anantharam ${ }^{1}$, Amit Sheth ${ }^{1}$, Rick L. Tarleton ${ }^{2}$

\author{
${ }^{1}$ Kno.e.sis Center, Wright State University; \\ ${ }^{2}$ Center for Tropical and Emerging Diseases, University of Georgia
}

ODBASE2009

Vilamoura, Algarve-Portugal

November 05, 2009 


\section{Parasite Research: Creation of Parasite Strains}
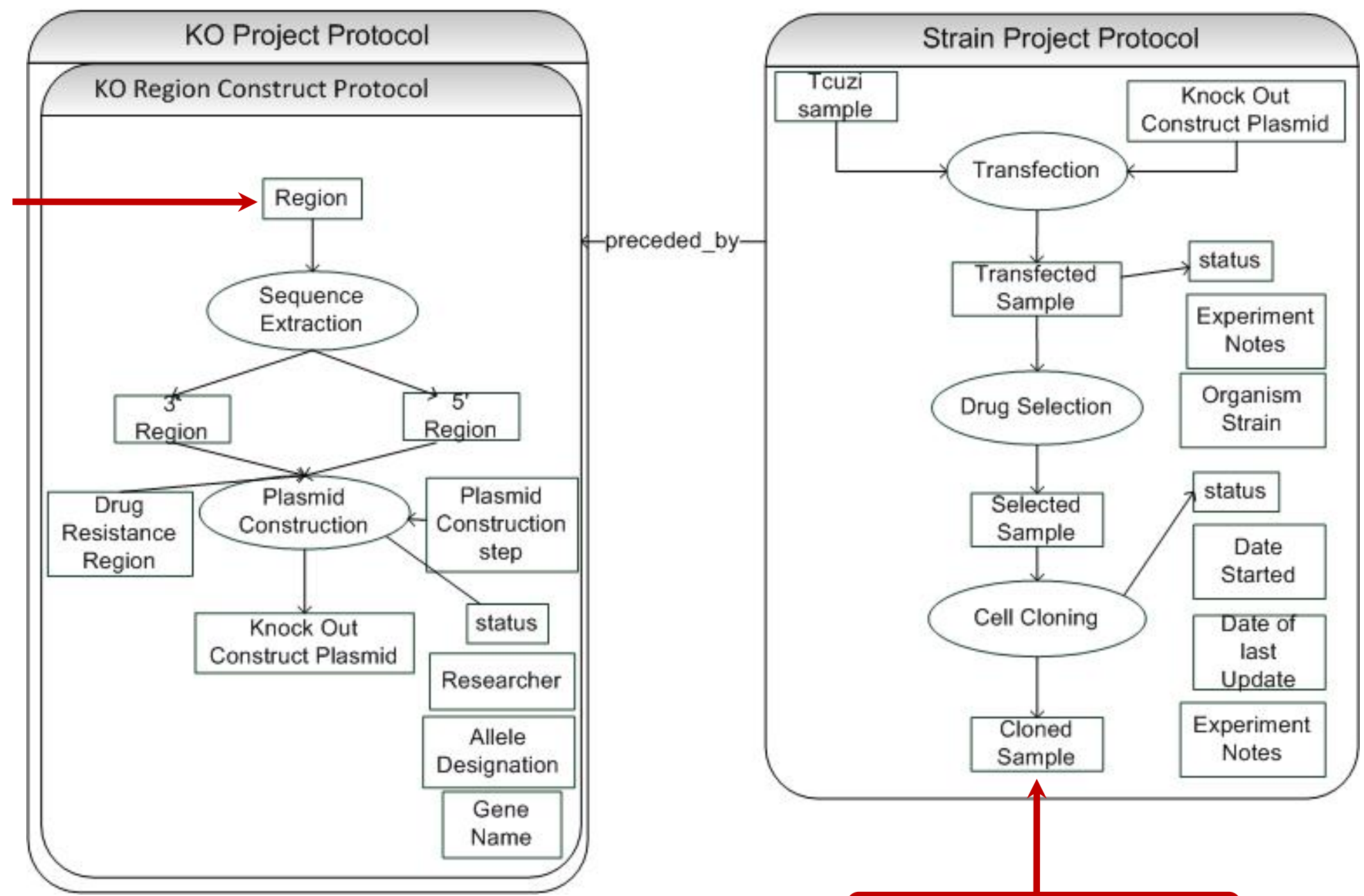


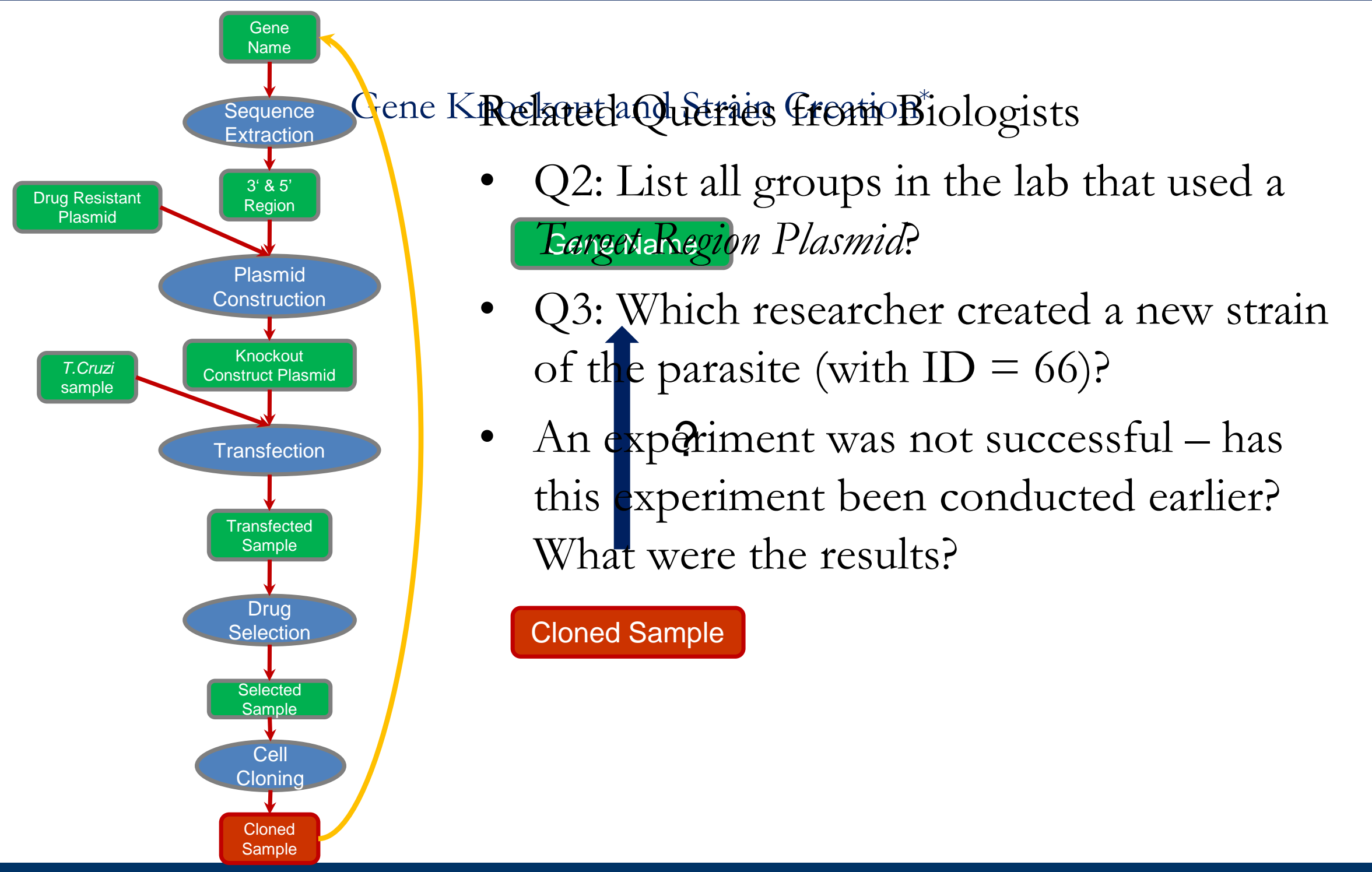




\section{Provenance Management in Science}

- Provenance from the French word "provenir" describes the lineage or history of a data entity

- For Verification and Validation of Data Integrity, Process Quality, and Trust

- Issues in Provenance Management

$>$ Provenance Modeling

$>$ A Dedicated Query Infrastructure

$>$ Practical Provenance Management Systems 


\section{Outline}

- Provenance Modeling: Provenir $\rightarrow$ Parasite Experiment ontology

- Provenance Query Infrastructure

- Provenance Query Engine

- Evaluation Results

- Query Optimization: Materialized Provenance Views 


\section{Ontologies for Provenance Modeling}

- Advantages of using Ontologies

> Formal Description: Machine Readability, Consistent Interpretation

$>$ Use Reasoning: Knowledge Discovery over Large Datasets

- Problem: A gigantic, monolithic Provenance Ontology! - not feasible

- Solution: Modular Approach using a Foundational Ontology

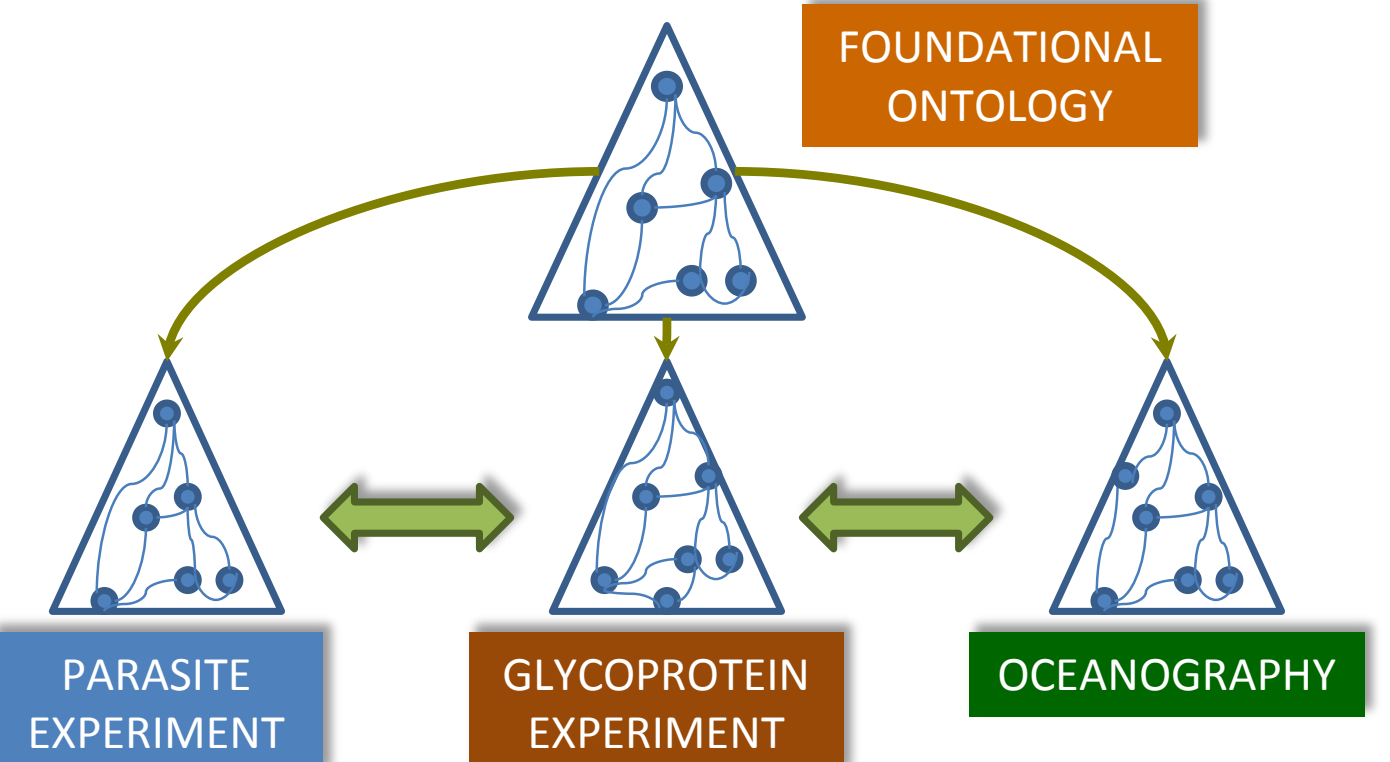




\section{Provenir Ontology}

\section{KnO.E.SIS}

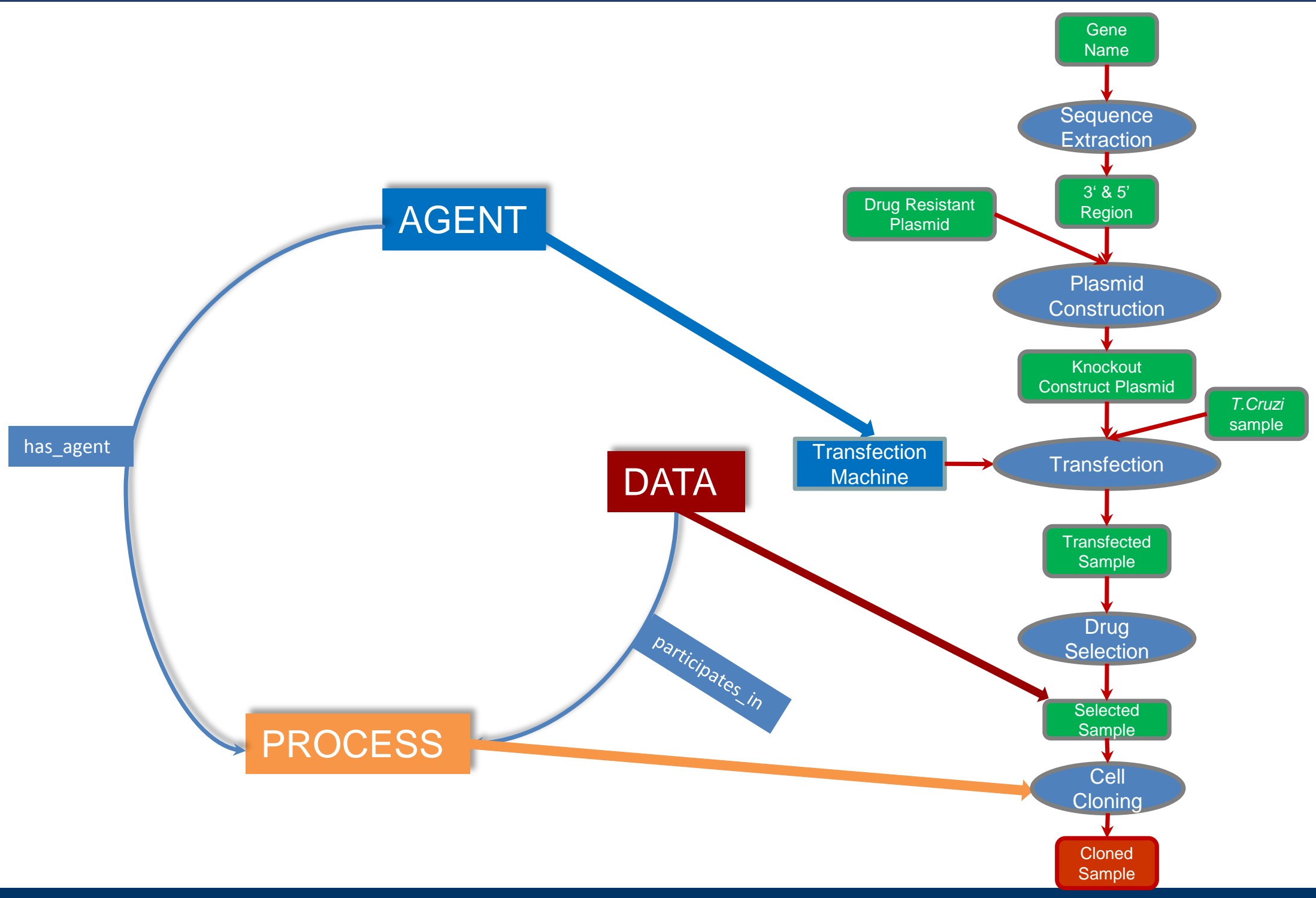




\section{Provenir Ontology Schema}

Kno.e.SIS
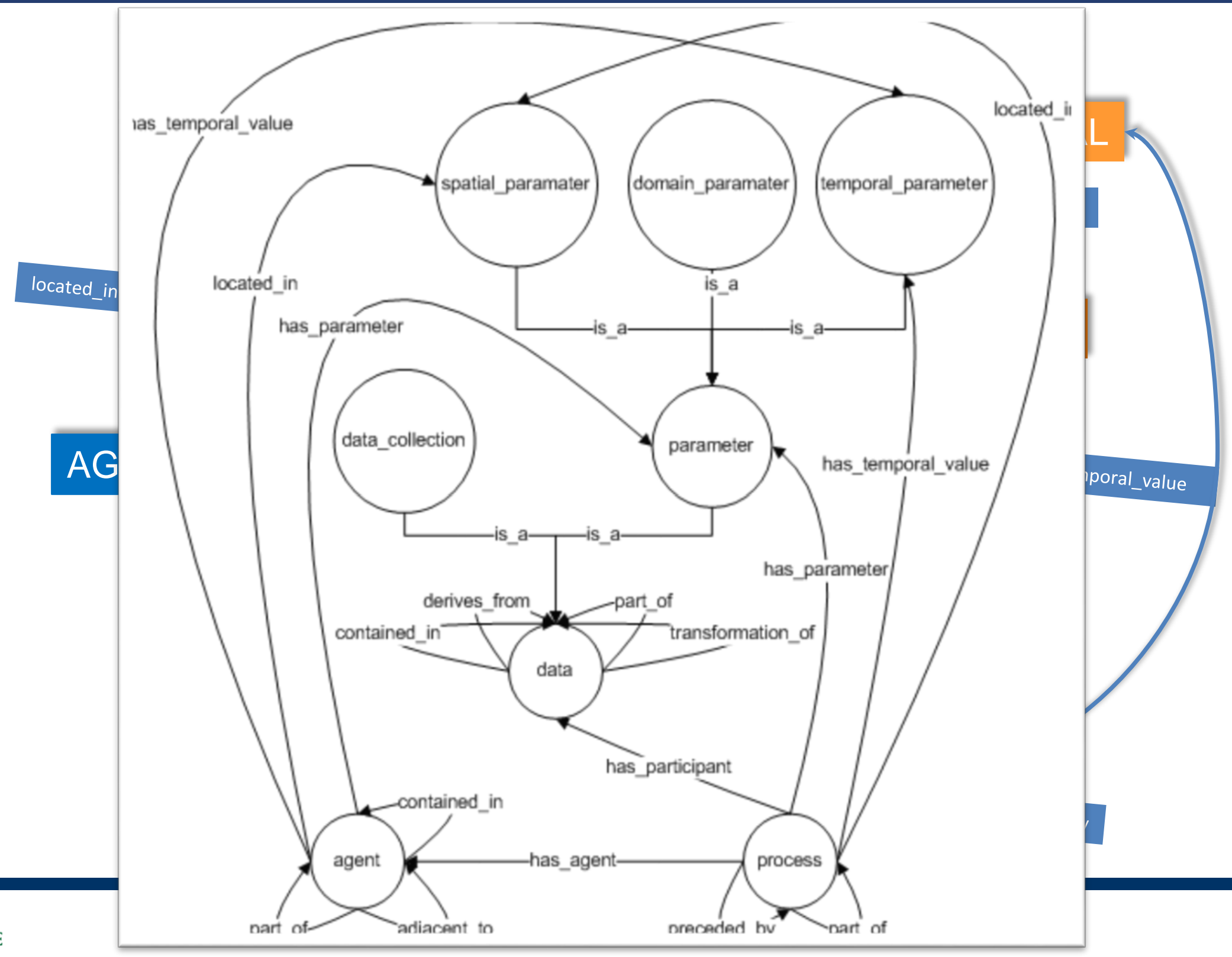
Domain-specific Provenance: Parasite Experiment ontology
KnO.E.SIS

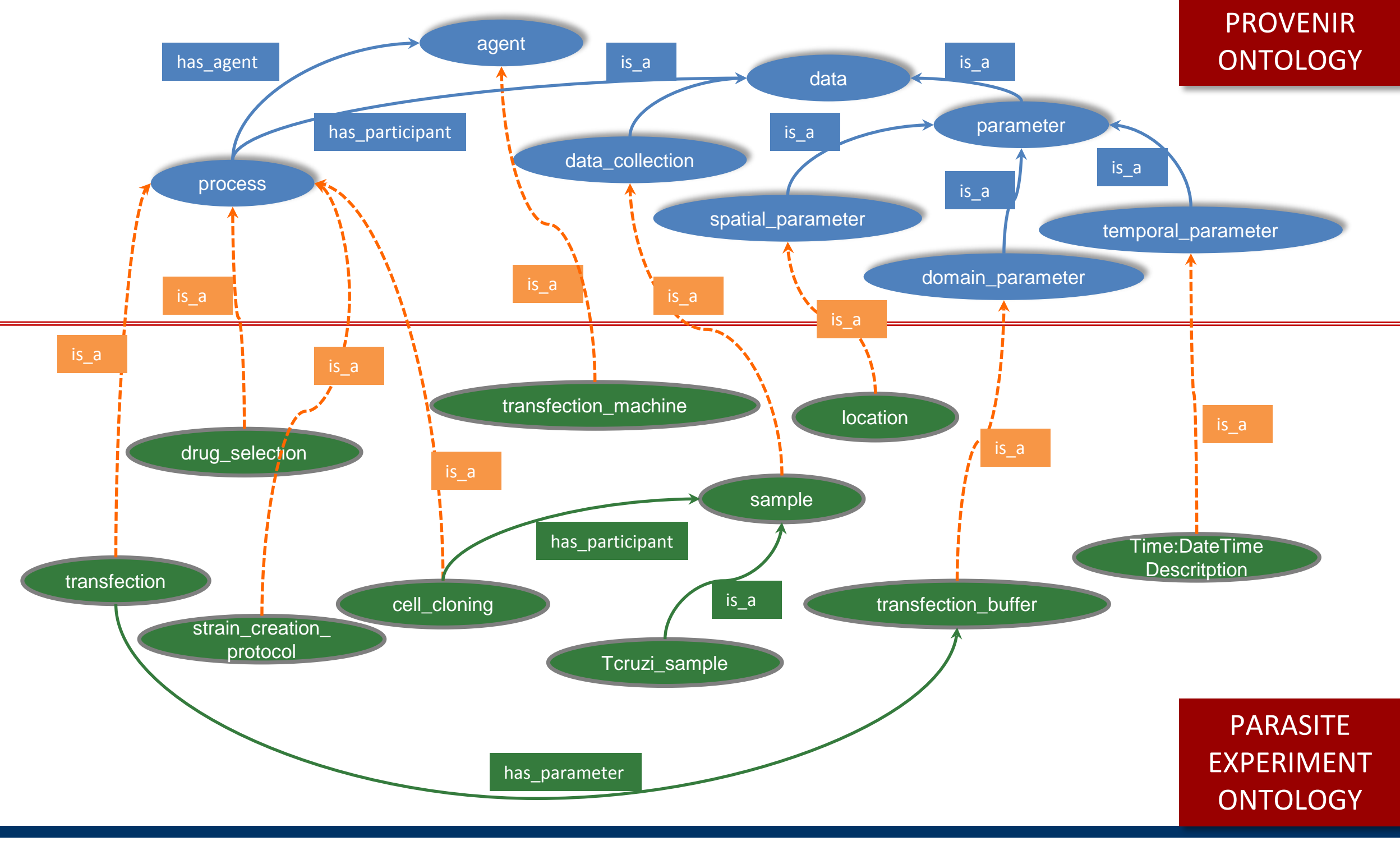

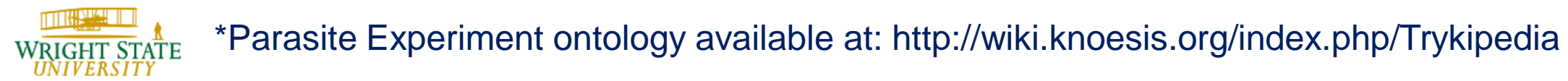




\section{Outline}

- Provenance Modeling: Provenir $\rightarrow$ Parasite Experiment ontology

- Provenance Query Infrastructure

- Provenance Query Engine

- Evaluation Results

- Query Optimization: Materialized Provenance Views 


\section{Provenance Query Classification}

Classified Provenance Queries into Three Categories

- Type 1: Querying for Provenance Metadata

o Example: Which gene was used create the cloned sample with ID = 66 ?

- Type 2: Querying for Specific Data Set

o Example: Find all knockout construct plasmids created by researcher Michelle using "Hygromycin" drug resistant plasmid between April 25, 2008 and August 15, 2008

- Type 3: Operations on Provenance Metadata

o Example: Were the two cloned samples 65 and 46 prepared under similar conditions - compare the associated provenance information 


\section{Provenance Query Operators}

Four Query Operators - based on Query Classification

- provenance ( -Closure operation, returns the complete set of provenance metadata for input data entity

- provenance_context 0 - Given set of constraints defined on provenance, retrieves datasets that satisfy constraints

- provenance_compare $($ - adapt the $\mathrm{RDF}$ graph equivalence definition

- provenance_merge $($ - Two sets of provenance information are combined using the RDF graph merge 


\section{Answering Provenance Queries using provenance O Operator}
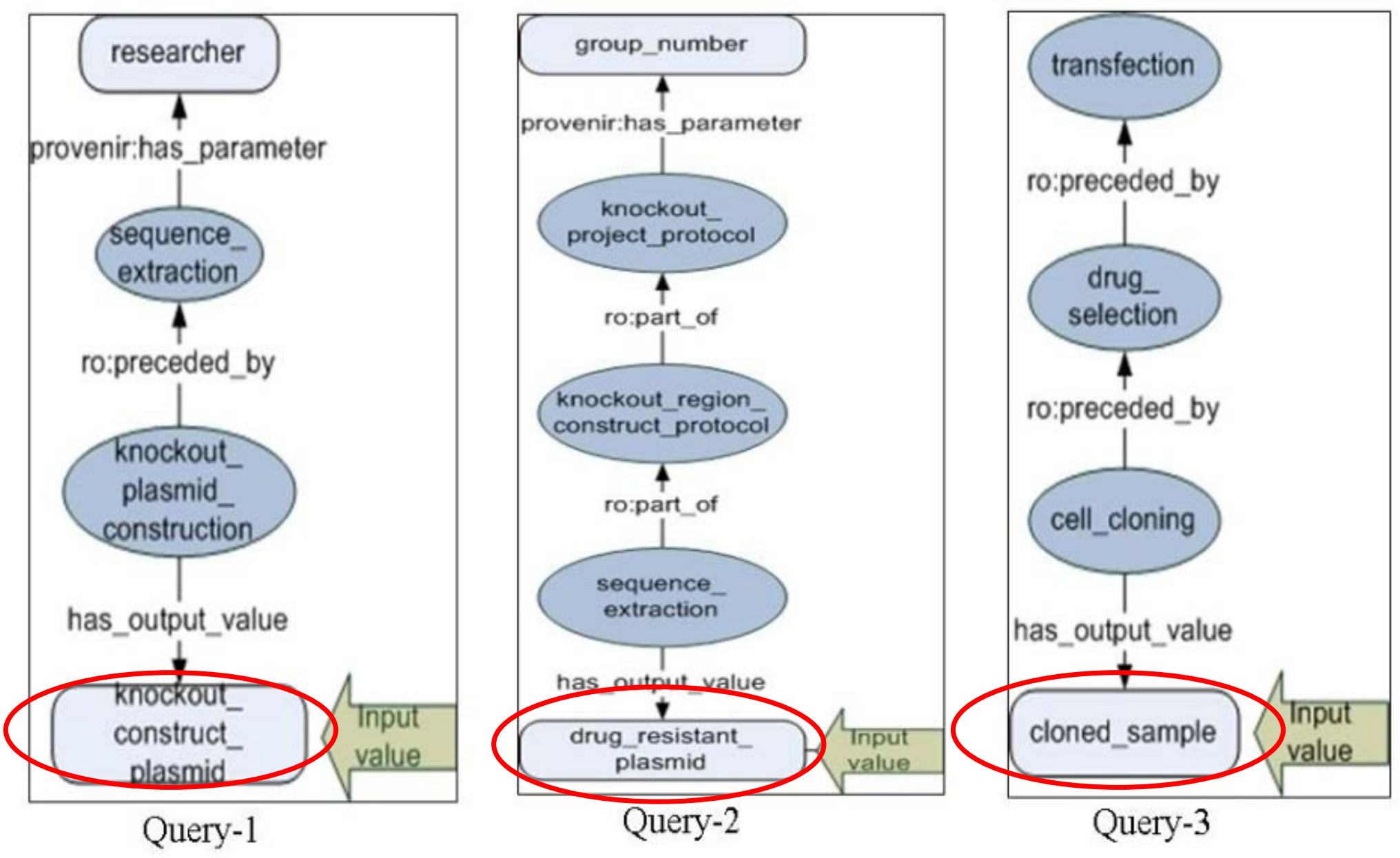


\section{Outline}

- Provenance Modeling: Provenir $\rightarrow$ Parasite Experiment ontology

- Provenance Query Infrastructure

- Provenance Query Engine

- Evaluation Results

- Query Optimization: Materialized Provenance Views 


\section{Provenance Query Engine}

- Available as API for integration with provenance management systems

- Layer on top of a RDF Data Store (Oracle 10g), requires support for:

0 Rule-based reasoning

o SPARQL query execution

- Input:

o Type of provenance query operator : provenance ()

O Input value to query operator: cloned sample 66

o User details to connect to underlying RDF store 


\section{Outline}

- Provenance Modeling: Provenir $\rightarrow$ Parasite Experiment ontology

- Provenance Query Infrastructure

- Provenance Query Engine

- Evaluation Results

- Query Optimization: Materialized Provenance Views 


\section{Evaluation Results}

- Queries expressed in SPARQL

- Datasets using real experiment data

\begin{tabular}{|c|c|c|c|}
\hline Query ID & $\begin{array}{l}\text { Number of } \\
\text { Variables }\end{array}$ & $\begin{array}{l}\text { Total } \\
\text { Number of } \\
\text { Triples }\end{array}$ & $\begin{array}{l}\text { Nesting } \\
\text { Levels using } \\
\text { OPTIONAL }\end{array}$ \\
\hline $\begin{array}{l}\text { Query 1: } \\
\text { Target plasmid }\end{array}$ & 25 & 84 & 4 \\
\hline $\begin{array}{l}\text { Query 2: } \\
\text { Plasmid_66 }\end{array}$ & 33 & 110 & 5 \\
\hline $\begin{array}{l}\text { Query 3: } \\
\text { Transfection } \\
\text { attempts }\end{array}$ & 67 & 190 & 7 \\
\hline $\begin{array}{l}\text { Query 4: } \\
\text { cloned_sample } \\
66\end{array}$ & 67 & 190 & 7 \\
\hline
\end{tabular}

\begin{tabular}{|l|r|r|}
\hline Dataset ID & $\begin{array}{l}\text { Number of } \\
\text { RDF } \\
\text { Inferred } \\
\text { Triples }\end{array}$ & $\begin{array}{l}\text { Total } \\
\text { Number of } \\
\text { RDF } \\
\text { Triples }\end{array}$ \\
\hline DS 1 & 2,673 & 3,553 \\
\hline DS 2 & 3,470 & 4,490 \\
\hline DS 3 & 4,988 & 6,288 \\
\hline DS 4 & 47,133 & 60,912 \\
\hline
\end{tabular}




\section{Evaluation Results}

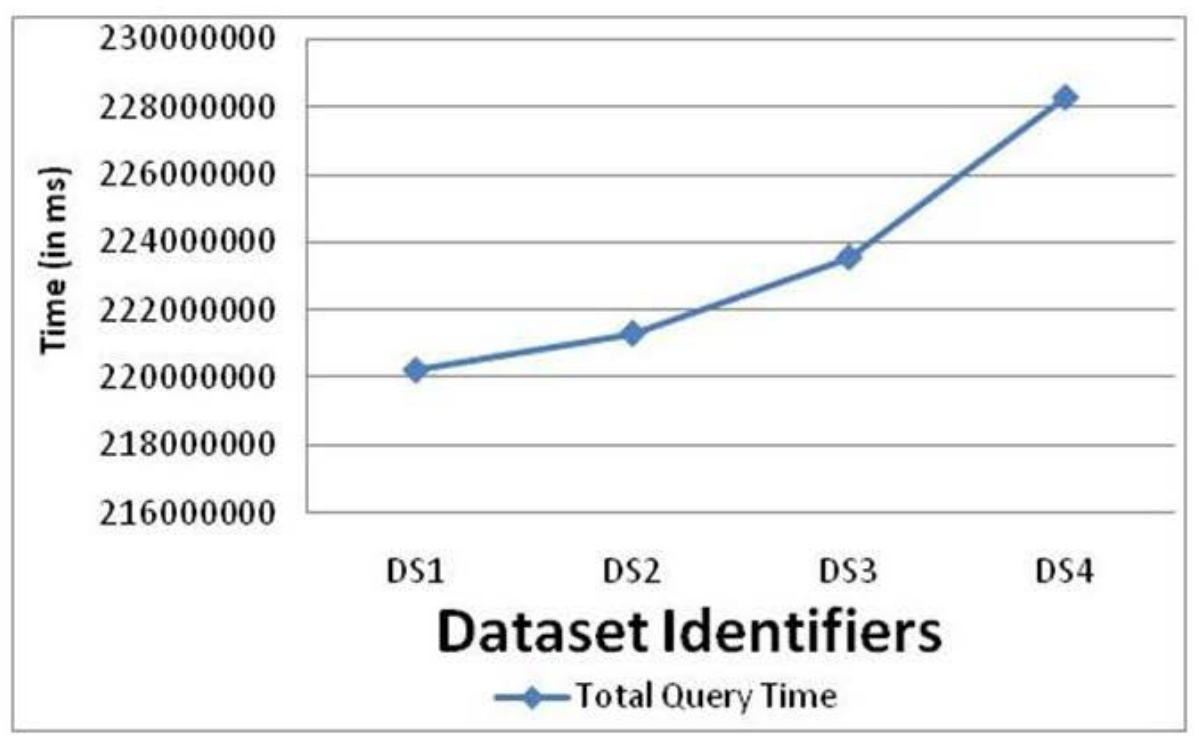

(a)

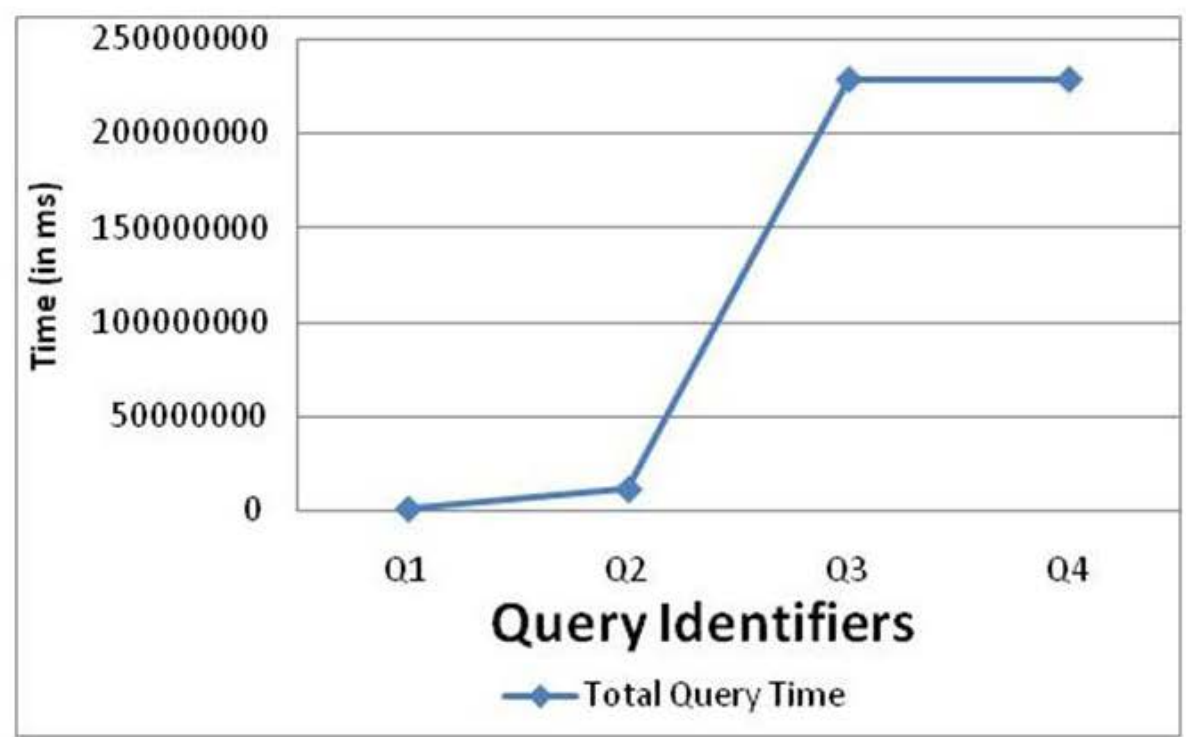

(b) 


\section{Outline}

- Provenance Modeling: Provenir $\rightarrow$ Parasite Experiment ontology

- Provenance Query Infrastructure

- Provenance Query Engine

- Evaluation Results

- Query Optimization: Materialized Provenance Views 


\section{Query Optimization: Materialized Provenance Views}

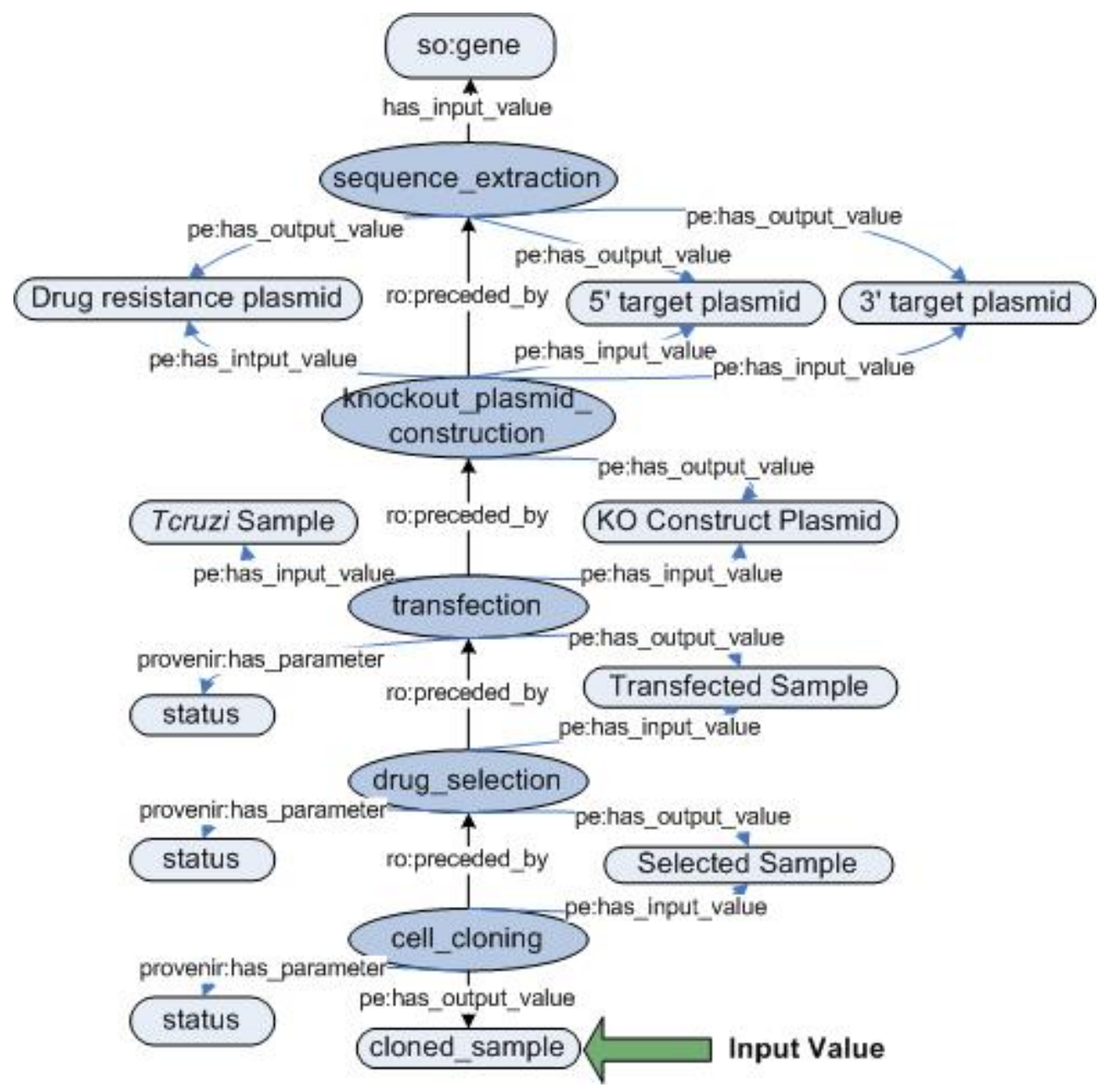

- Materializes a single logical unit of provenance

- Does not require queryrewriting

- View updates: addressed by characteristics of provenance

- Created using a memoization approach 


\section{Provenance Query Engine Architecture}

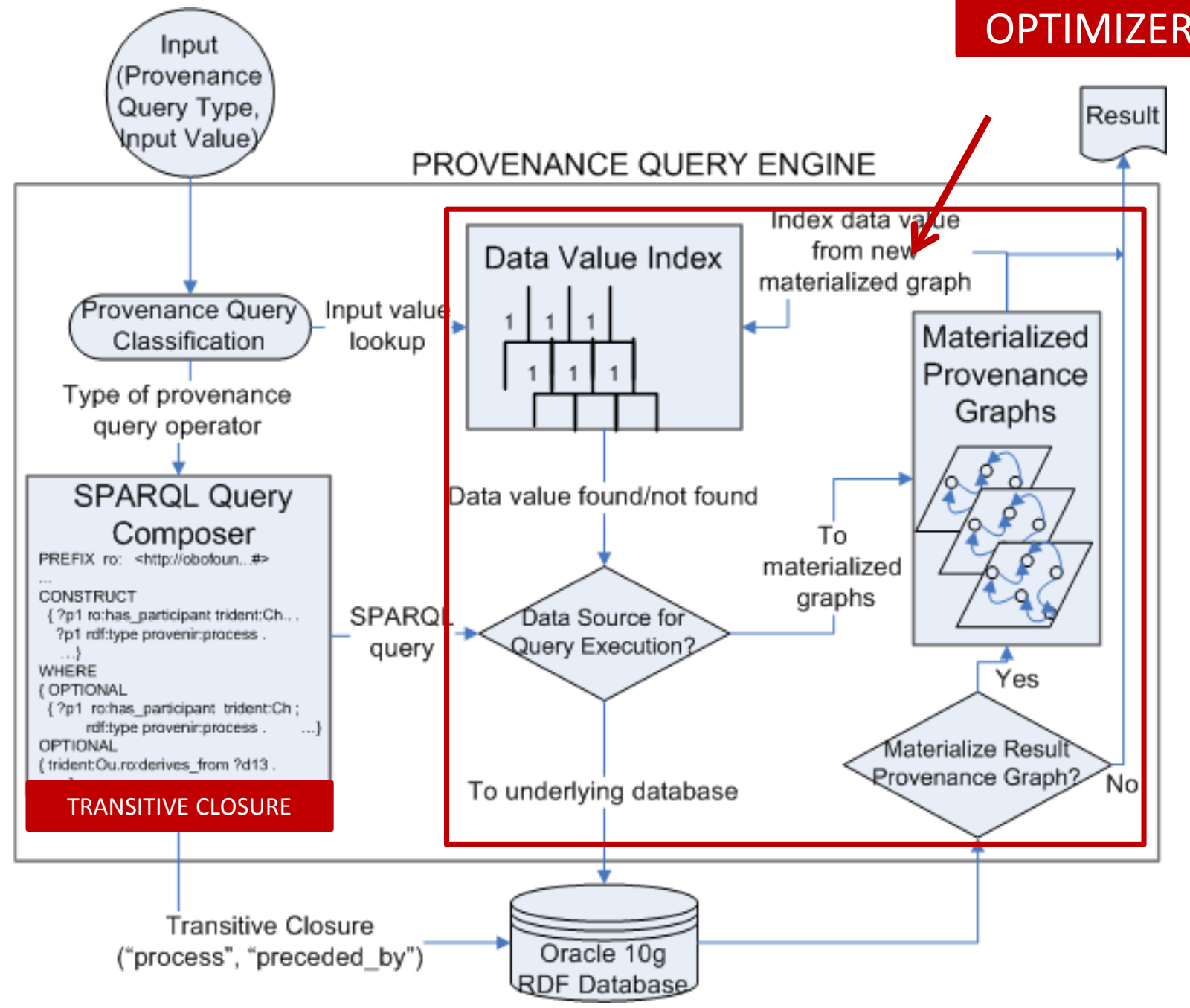




\section{Evaluation Results using Materialized Provenance Views}

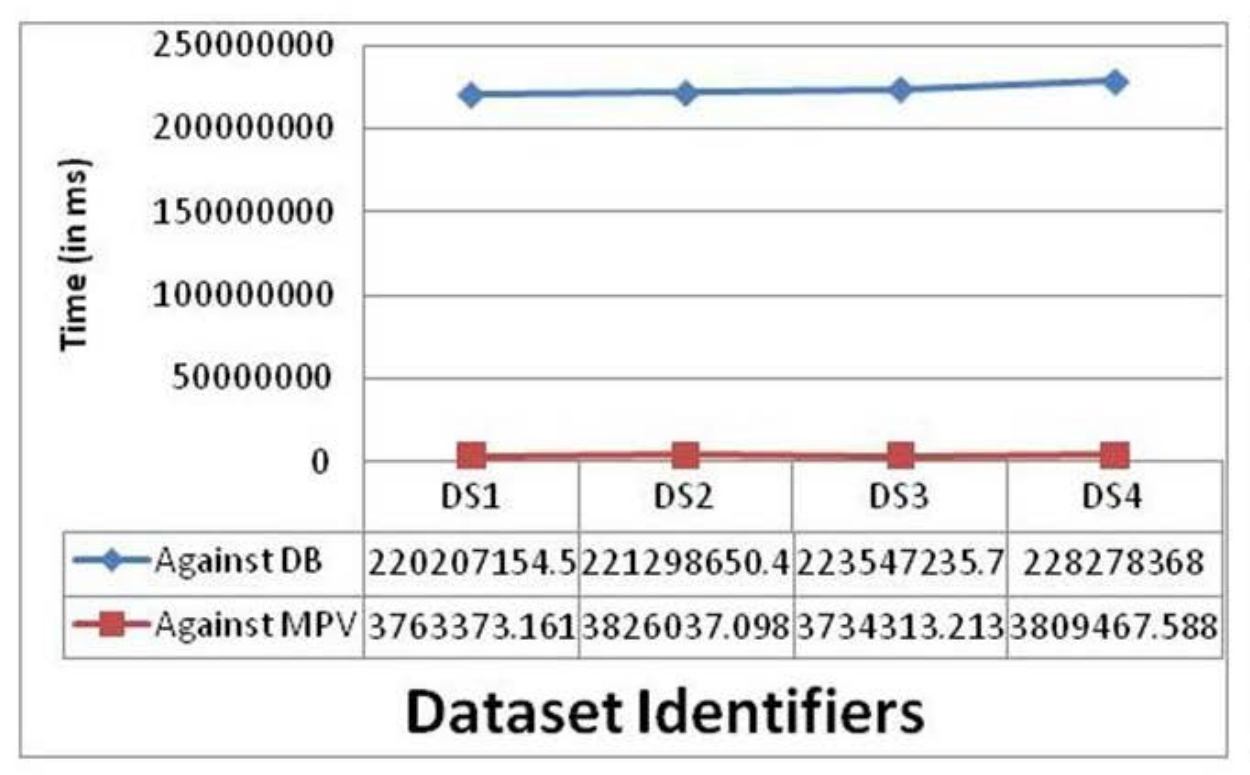

(a)

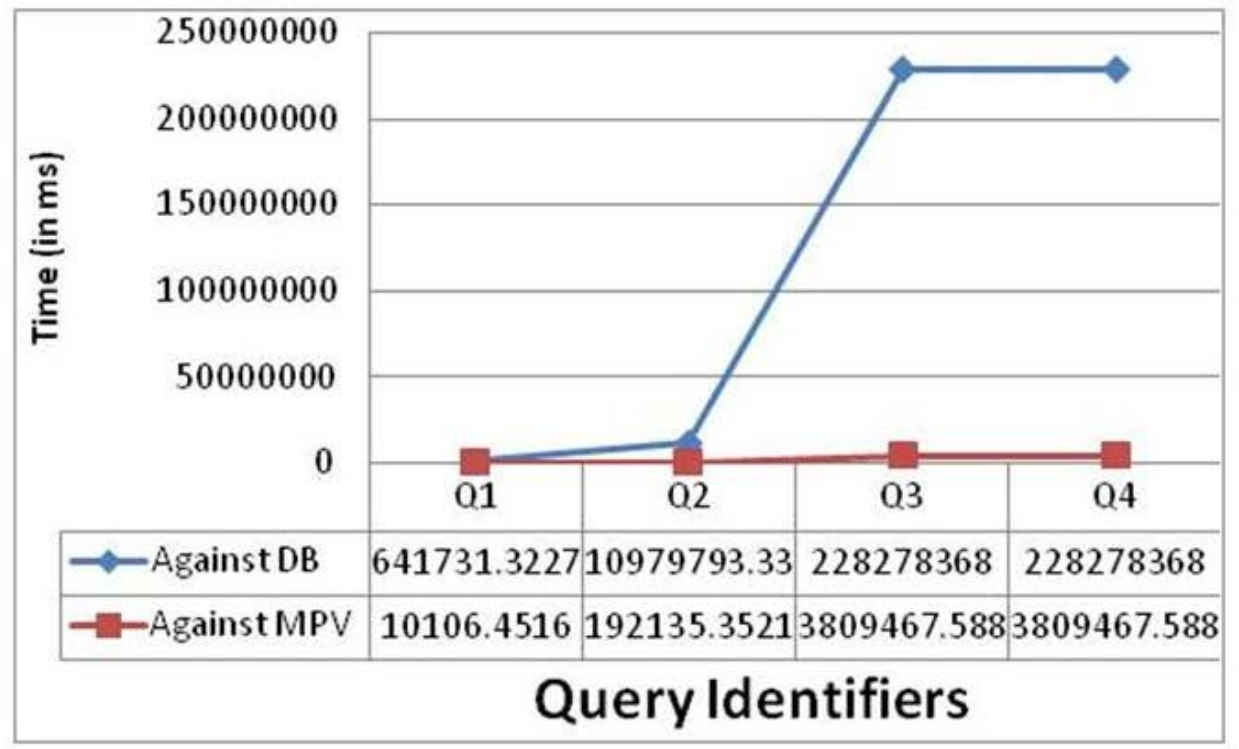

(b) 


\section{Provenance Management System for Parasite Research}

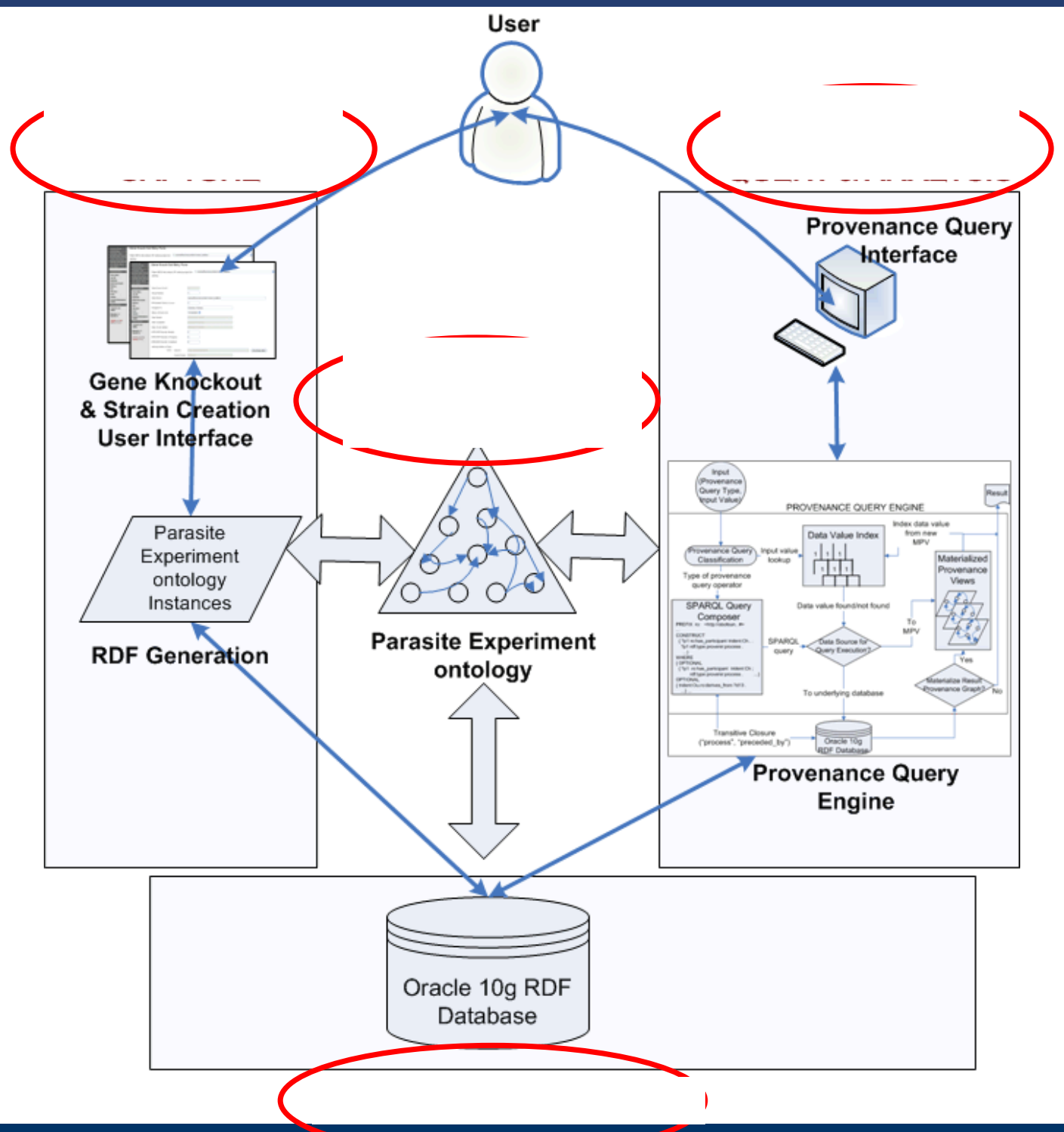




\section{Acknowledgement}

\section{Kno.e.SIS}

- Flora Logan - The Wellcome Trust Sanger Institute, Cambridge, UK

- Priti Parikh - Kno.e.sis Center, Wright State University

- Roger Barga - Microsoft Research, Redmond

- Jonathan Goldstein - Microsoft Research, Redmond 
Contact email: satyasahoo@gmail.com Web:

http://knoesis.wright.edu/researchers/satya/ 